\title{
Oral administration of bovine lactoferrin attenuates ultraviolet B-induced skin photodamage in hairless mice
}

\author{
M. Murata, ${ }^{*}{ }^{1}$ T. Satoh, ${ }^{*}$ H. Wakabayashi, ${ }^{\star}$ K. Yamauchi, ${ }^{*}$ F. Abe, ${ }^{*}$ and Y. Nomura† \\ ${ }^{*}$ Food Science and Technology Institute, Morinaga Milk Industry Co. Ltd., Zama-City, Kanagawa 252-8583, Japan \\ †Applied Protein Chemistry, Faculty of Agriculture, Tokyo University of Agriculture and Technology, Fuchu-City, Tokyo 183-8509, Japan
}

\begin{abstract}
Lactoferrin (LF) is recognized as a host defensive glycoprotein, especially for newborn infants. The aim of this study was to investigate whether orally administered LF had protective activity against UV-induced skin damage in hairless mice. Transepidermal water loss and skin hydration were evaluated in nonirradiated mice, UVB-irradiated mice, and UVB-irradiated and LF-administered mice. Supplementation with LF (1,600 $\mathrm{mg} / \mathrm{kg}$ per day) effectively suppressed the increase in transepidermal water loss, reduction in skin hydration, aberrant epidermal hyperplasia, and cell apoptosis induced by UV irradiation. Although no significant changes in superoxide dismutase-like activity or malondialdehyde levels were observed in the skin with both UV irradiation and LF administration, UV-stimulated IL-1 $\beta$ levels in the skin were significantly suppressed by the administration of LF. Oral supplementation with LF has the potential to reduce IL-1 $\beta$ levels and prevent UV-induced skin damage. Further studies are needed to elucidate the relationships between the antiinflammatory effects and skin protective function of LF.

Key words: lactoferrin, UV-induced skin damage, antiinflammatory effect, hairless mice
\end{abstract}

\section{INTRODUCTION}

The epidermis is a physiological barrier that protects the organism against pathogens and chemical or physiological damage. Exposure to UV radiation is the most important environmental factor associated with skin damage, which affects the structure and function of the skin throughout life. Solar UV radiation often induces photochemical skin changes that may lead to acute effects such as erythema or sunburn, or to chronic effects including photoaging and skin tumors (Berneburg et al., 1997). Aging-induced skin changes have a negative effect on a person's quality of life. Thus, there has been

Received June 18, 2013.

Accepted November 3, 2013.

${ }^{1}$ Corresponding author: m-murata@morinagamilk.co.jp great interest in the prevention of skin aging though cosmetics and functional foods (Yaar et al., 2002). Skin damage is initiated by the photochemical generation of reactive oxygen species (ROS). Ultraviolet-induced ROS also cause direct chemical modifications to cellular components in the skin, including DNA, proteins, and lipids (Meffert et al., 1976; Sander et al., 2002). Previous studies have shown that collagen synthesis is decreased by UV irradiation and is a major factor in extrinsic skin aging (Fisher et al., 2000; Quan et al., 2004).

Lactoferrin ( $\mathbf{L F})$ is an $80-\mathrm{kDa}$ iron-binding glycoprotein of the transferrrin family, which was first discovered in cow milk, but is also abundant in other body fluids such as colostrum, saliva, and tears in most mammals (Baker, 2005; Legrand, 2012). Lactoferrin is a key element in the host defense system and is known to have various biological functions, including antimicrobial, antiviral, and antiinflammatory activities, and prevention of oxidation (Bellamy et al., 1992; Yamauchi et al., 1998; Actor et al., 2009; Kuhara et al., 2012). Previous in vitro studies have suggested that bovine LF or its derived peptide play a protective role against UV-induced DNA damage in corneal epithelial cells (Shimmura et al., 1996; Fujihara et al., 2000) and human colon cells (Freiburghaus et al., 2012).

Recent studies have reported that human LF directly stimulates fibroblast and keratinocyte function, which may support skin wound healing (Takayama and Takezawa, 2006; Tang et al., 2010a,b; Takayama and Aoki, 2012). Bovine LF has also been suggested to promote hyaluronan and collagen synthesis in fibroblasts in an in vitro study (Saito et al., 2011). As for collagen metabolism, lactoferricin, an isolated active peptide of LF-hydrolysate, is shown to have antiinflammatory and anticatabolic activities against collagen degradation in human articular cartilage and synovium fibroblasts (Yan et al., 2013). Although in vitro studies investigating the mechanisms of LF on keratinocytes and fibroblasts have been performed, the precise action and mechanism of LF in vivo are not fully understood. To date, the effects of administered LF on UV-irradiated skin have yet to be thoroughly investigated. 
In this study, we investigated whether LF supplementation had a protective function against UV-induced skin aging, including morphological changes and skinbarrier damage, in hairless mice.

\section{MATERIALS AND METHODS}

\section{Animals}

All animal experiments were approved by the Animal Research Committee of Morinaga Milk Industry (Kanagawa, Japan). Seven-week-old male Hos:HR-1 hairless mice (Japan SLC, Shizuoka, Japan) were housed in a single cage at $22 \pm 2^{\circ} \mathrm{C}$ on a 12-h light and dark cycle, with free access to water and a Lab MR stock diet (Nosan, Tokyo, Japan). Following 2 wk of acclimation, mice were divided into 3 groups $(8$ mice per group) such that BW, hydration of the stratum corneum, and transepidermal water loss (TEWL) were not significantly different among the groups: nonirradiated control mice $[\mathbf{U V}(-)$ Saline], UV-irradiated control mice $[\mathbf{U V}(+)$ Saline], and UV-irradiated and LF-administered mice $[\mathbf{U V}(+) \mathbf{L F}]$. Body weight, food intake, and water intake were monitored every week throughout the experimental period. Mice fasted for 18 $\mathrm{h}$ before being killed under inhalation anesthesia.

\section{Sample Administration}

Bovine LF (Morinaga Milk Industry) was dissolved in saline $(0.9 \% \mathrm{NaCl})$ and continuously administered per os with a feeding tube at 1,600 mg/ $\mathrm{kg}$ of BW for 6 $\mathrm{d}$ a week. The same volume of vehicle (saline) was used as a control.

\section{UVB Irradiation}

Irradiation with UVB followed the method described in a previous report (Tanaka et al., 2009). A mouse was housed in a stainless steel cage and subjected to UVB irradiation $\left(0.9 \mathrm{~mW} / \mathrm{cm}^{2}\right)$ emitted from UVB lamps with peak emission at $306 \mathrm{~nm}$ (GL20E; Sankyo Denki, Tokyo, Japan). Irradiation was performed 3 times a week. To avoid the generation of acute erythema, UVB irradiation was started at one-half of the minimum erythema dose and increased gradually each week. The intensity of UVB was monitored using the Dermaray UV meter DMR-UV-M-2 (Terumo, Tokyo, Japan). The total energy of UVB that each mouse received was 3.0 $\mathrm{J} / \mathrm{cm}^{2}$ over $7 \mathrm{wk}$.

\section{TEWL and Hydration of the Stratum Corneum}

Skin barrier function in the mouse was evaluated by TEWL. The TEWL of the stratum corneum in the dorsal skin was measured once a week with a Tewameter TM 300 (Courage + Khazaka Electronic, Köln, Germany) after acclimation at $22 \pm 2^{\circ} \mathrm{C}$ and $50 \pm 5 \%$ humidity. The average TEWL value for $5 \mathrm{~s}$ under stable conditions was adopted as the average value for the mouse. Hydration of the stratum corneum in the dorsal skin was measured with a Corneometer CM 825 (Courage + Khazaka Electronic) under the same conditions. Hydration units of the stratum corneum were calculated as the average of more than 10 measurement values.

\section{Anatomy, Sampling, and Histology}

After the $7 \mathrm{wk}$ of experimental period, mice were killed under inhalation anesthesia of Sevofluran (Sevoflo, DS Pharma Animal Health, Osaka, Japan), and serum and central dorsal skin samples were obtained. Skin samples were subjected to histology and protein extraction. Serum samples were prepared by centrifugation at $1,000 \times g$ for $10 \mathrm{~min}$.

Skin samples $\left(10 \mathrm{~mm}^{2}\right)$ were fixed in Bouin's solution (Wako, Osaka, Japan), and embedded in paraffin after substitution with ethanol. The block was sliced into $3-\mu \mathrm{m}$ sections. These sections were then stained with hematoxylin and eosin. The stained skin samples were observed by microscope BX53 (Olympus, Tokyo, Japan). Epidermal thickness was analyzed by CellSens Dimension software (Olympus), and the average thickness of 30 points per mouse was adopted as the average epidermal thickness.

\section{Superoxide Dismutase-Like Activity}

Dorsal skin samples were obtained with disposable biopsy punches (Kai Industries, Gifu, Japan), and homogenized using a Polytron homogenizer (Kinematica, Lucerme, Switzerland) in Tris-EDTA buffer $(10 \mathrm{mM}$ Tris- $\mathrm{HCl}, 1 \mathrm{~m} M$ EDTA, $0.25 M$ sucrose, $\mathrm{pH}$ 7.4) on ice. To prepare the skin homogenate suspension, the homogenate solution was centrifuged at $15,800 \times g$ for $60 \mathrm{~min}$ at $4^{\circ} \mathrm{C}$. The suspension was subjected to the superoxide dismutase (SOD)-like activity test using the SOD Assay kit WST (Dojindo, Kumamoto, Japan). The SOD-like activity was assayed and calculated as the $50 \%$ inhibitory concentration $\left(\mathrm{IC}_{50}\right)$ of soluble formazan formation per skin weight.

\section{Quantitative Analysis of Malondialdehyde and Cytokines}

Dorsal skin was sampled using the same procedure as the SOD assay described above and was homogenized in $50 \mathrm{mM}$ phosphate buffer ( $\mathrm{pH}$ 7.2) including butylated hydroxytoluene. The supernatant of the homog- 
enate was collected after centrifugation at $1,000 \times g$ for $60 \mathrm{~min}$, and subjected to the malondialdehyde (MDA) assay using the MDA assay kit (Percipio Biosciences, Burlingame, CA).

Inflammatory cytokine [IL-1 $\beta$, IL-6, and tumor necrosis factor- $\alpha$ (TNF- $\alpha)$ ] levels in the serum and supernatant of skin homogenate samples were determined by the Quantikine ELISA kit (R\&D Systems, Minneapolis, MN). The supernatant of skin homogenate samples was prepared under the same conditions as samples for SOD-like activity. Interleukin-1 $\beta$ levels in serum samples were also determined by the Quantikine ELISA kit (R\&D Systems).

\section{Statistical Analysis}

Data are presented as the mean \pm standard error of mean (SEM). Statistical analysis was performed using Tukey-Kramer's multiple comparison tests. $P$-values $<$ 0.05 were considered significant.

\section{RESULTS}

\section{Animals}

Throughout the experimental period, BW, food intake, and the amount of drinking did not differ significantly among the treatment groups (data not shown).

\section{Effect of LF Administration on the Skin-Barrier Function}

Transepidermal water loss and hydration were measured to determine whether the water barrier was affected by UV irradiation and administration of LF. As shown in Figure 1, TEWL in the UV $(+)$ Saline group and $\mathrm{UV}(+)$ LF group started to increase from 4 wk. After 5 to 6 wk of irradiation, TEWL in the UV $(+)$ Saline group continued to increase to $22.4 \pm 2.3 \mathrm{~g} / \mathrm{m}^{2} \cdot \mathrm{h}$. In contrast, the increase in TEWL in the UV $(+) \mathrm{LF}$ group was suppressed to $13.1 \pm 1.8 \mathrm{~g} / \mathrm{m}^{2} \cdot \mathrm{h}[P<0.05$ vs. UV(+) Saline], and TEWL in the UV(-) Saline group remained stable $\left(4.3-6.0 \mathrm{~g} / \mathrm{m}^{2} \cdot \mathrm{h}\right)$.

Corresponding to the increase in TEWL, skin hydration in the UV(+) Saline and UV(+) LF groups started to decrease from 4 wk (Figure 2). However, skin hydration was maintained in the $\mathrm{UV}(+) \mathrm{LF}$ group relative to that the UV(+) Saline group during wk 5 to 7 .

\section{Effect of LF Administration on Histological Changes}

Figure 3 shows a representative image of a mouse skin sample from each group, visualized using hematoxylin and eosin $(\mathrm{H} \& \mathrm{E})$ staining. Epidermal thickening was observed in both the UV $(+)$ Saline group and the $\mathrm{UV}(+)$ LF group; however, it was significantly greater in the UV $(+)$ Saline group $(P<0.05)$. Furthermore, in the $U V(+)$ Saline group, a large number of multilayered and apoptotic epithelium cells were apparent above the basement membrane. In contrast, a small number of multilayered and apoptotic epithelium cells were observed in the $\mathrm{UV}(+) \mathrm{LF}$ group.

Figure 4 shows quantitative epidermal thickness analyzed using analyzed using CellSens Dimension software (Olympus). In the $\mathrm{UV}(+)$ Saline group, epidermal thickness increased significantly to 50 to $60 \mu \mathrm{m}(P<$ 0.05), whereas epidermal thickening in the $\mathrm{UV}(+) \mathrm{LF}$ group was significantly suppressed to around $25 \mu \mathrm{m}(P$ $<0.05)$.

\section{Effect of LF Administration on ROS-Related Parameters}

Oxidative modifications to skin proteins induce ROS, which may be one of the key factors in skin damage. To investigate the effect of LF, we analyzed oxygen-related parameters in the skin of UV-irradiated

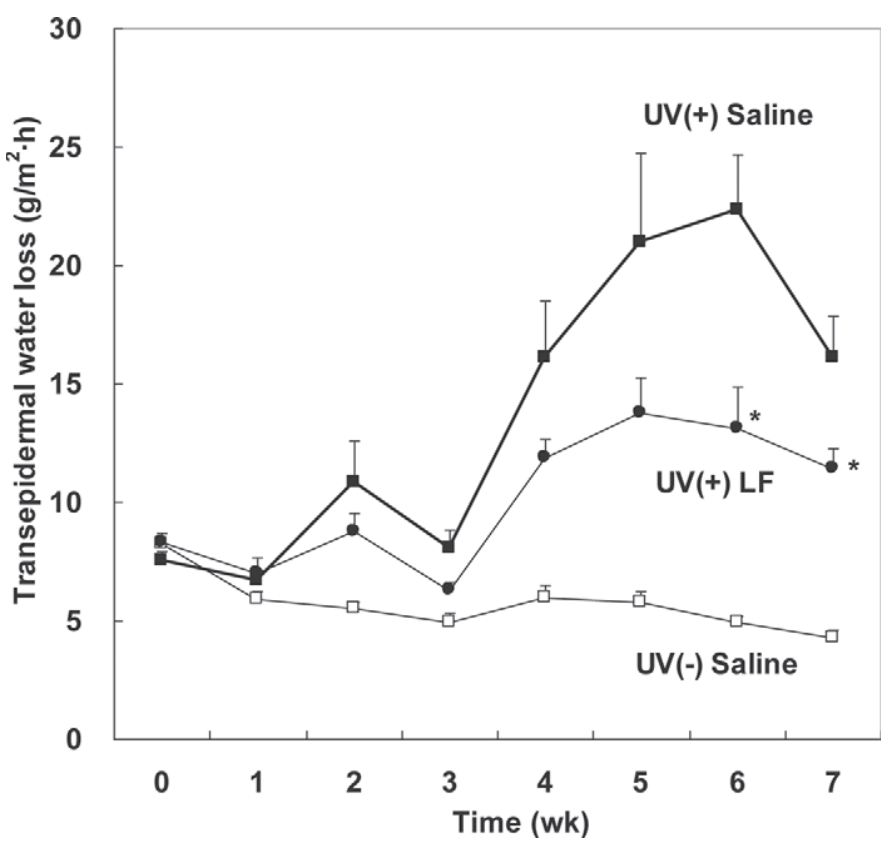

Figure 1. Effect of UVB irradiation and lactoferrin (LF) administration on the skin-barrier function of hairless mice. Transepidermal water loss (TEWL) in the stratum corneum was evaluated in nonirradiated mice $[\square$; UV $(-)$ Saline], UVB-irradiated mice $[\mathbf{\square}$; UV $(+)$ Saline], and UVB-irradiated and LF-administered mice [ $\bullet$ : UV $(+) \mathrm{LF}]$ at 1 -wk intervals. Data are presented as the mean \pm SEM of 8 mice in each group, before the experiment ( $0 \mathrm{wk})$ and 1 to $7 \mathrm{wk}$ after UVB irradiation. ${ }^{*} P<0.05$ : significant difference between the UV(+) Saline group and $\mathrm{UV}(+)$ LF group. 


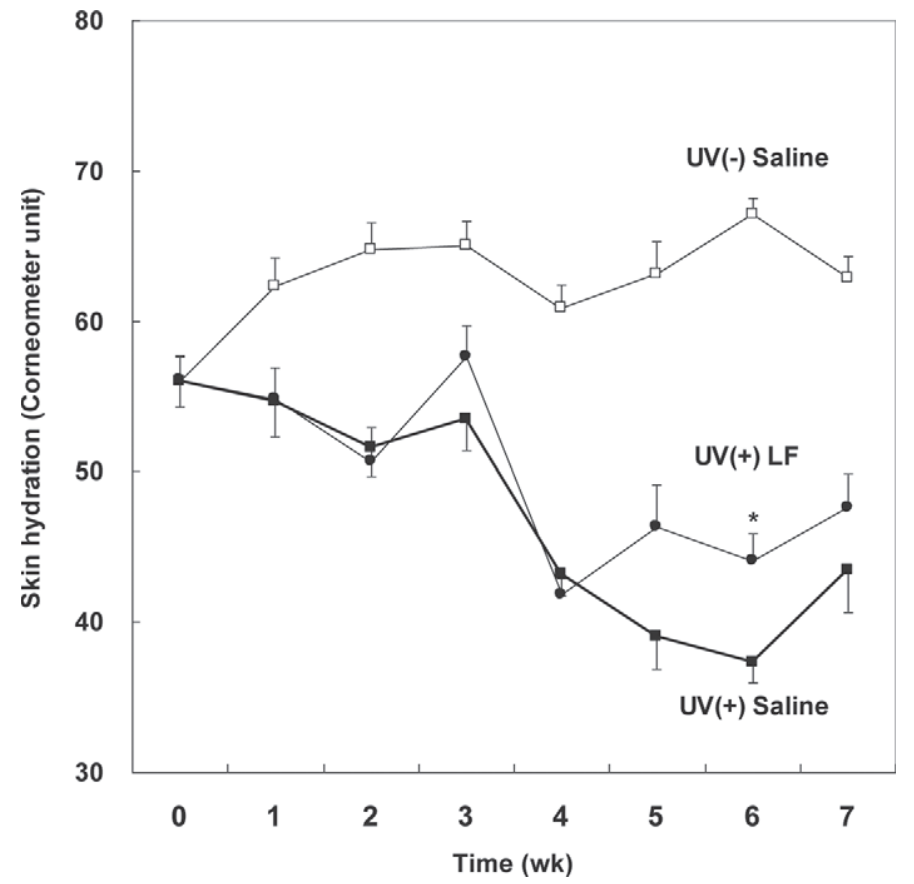

Figure 2. Effect of UVB irradiation and lactoferrin (LF) administration on skin hydration in hairless mice. Hydration units of the stratum corneum were evaluated in nonirradiated mice $[\square$; UV $(-)$ Saline], UVB-irradiated mice [ $\mathbf{\square}$; UV $(+)$ Saline], and UVB-irradiated and LF-administered mice $[\mathbf{0}$ UV $(+) \mathrm{LF}]$ at 1 -wk intervals. Data are presented as the mean \pm SEM of 8 mice in each group before the experiment $(0 \mathrm{wk})$ and 1 to $7 \mathrm{wk}$ after UVB irradiation. ${ }^{*} P<0.05$ : significant difference between the UV $(+)$ Saline group and $U V(+)$ LF group.

hairless mice. As shown in Figure 5A, SOD-like activity was lower in the $\mathrm{UV}(+)$ Saline group than in the $\mathrm{UV}(-)$ Saline group; however, SOD-like activity in the $\mathrm{UV}(+)$ LF group was not increased. Levels of MDA in the skin were not significantly different between the $\mathrm{UV}(-)$ Saline and UV(+) Saline groups, as shown in Figure 5B.

\section{Effect of LF Administration on IL-1 $1 \beta$ Levels}

In previous studies, UV irradiation was shown to activate nuclear factor (NF)- $\kappa \mathrm{B}$, which induce the expression of proinflammatory cytokines such as IL-1 $1 \beta$, IL-6, and TNF- $\alpha$, leading to stimulation of the expression of matrix metalloproteinases (Wan et al., 2001). Thus, we investigated inflammatory cytokine levels, including IL-1 $\beta$, IL-6, and TNF- $\alpha$, in UV-irradiated skin. As shown in Figure 6A, although IL-1 $\beta$ levels in the $\mathrm{UV}(+)$ Saline group were higher than those in the $\mathrm{UV}(-)$ Saline group, IL-1 $\beta$ levels in the UV $(+)$ LF group were significantly lower than those in the UV $(+)$ Saline group. Levels of IL-6 and TNF- $\alpha$ in the skin were too low and we could not obtain quantitative data (data not shown). We then analyzed IL-1 $\beta$ levels in the serum using the same procedure as that in the skin. As shown in Figure 6B, IL-1 $\beta$ levels in the serum did not vary significantly among the UV(-) Saline, UV $(+)$ Saline, and UV $(+)$ LF groups.

\section{DISCUSSION}

Chronic exposure to UVB irradiation is known to damage skin structure and function, as characterized by wrinkles, laxity, roughness, and irregular pigmentation. A photodamage model was developed by applying repeated low-dose UVB irradiation to the skin of hairless mice (Kligman et al., 1982). Several in vivo studies have been carried out using this photodamage model for qualitative and quantitative changes resembling the damage that occurs to human skin (Schwartz, 1988; Kiss et al., 1991; Zheng and Kligman, 1993).

Ultraviolet irradiation is considered to generate ROS, which have a negative effect on the skin, including increases in lipid peroxide levels and DNA damage. Previous studies showed that activity levels of antioxidant molecules such as SOD, catalase, and glutathione peroxidase, which play important roles in protection against photodamage, changed after acute and chronic UV irradiation (Pence and Naylor, 1990; Iizawa et al., 1994; Okada et al., 1994; Shindo et al., 1994).

Ultraviolet irradiation to the skin has also been shown to stimulate the production of inflammatory cytokines (IL-1 $\beta$, IL-6, and TNF- $\alpha$ ) as an early stage of wound healing and recruit inflammatory leukocytes. Furthermore, these inflammatory cytokines induce collagenase and the synthesis of other proteinases, including matrix metalloproteinases, through the activation of nuclear factor activator protein-1 (Fisher et al., 2002; Rittié and Fisher, 2002).

We observed sequential UV-irradiated skin changes using TEWL and hydration as skin property indices. We confirmed that skin-barrier function was gradually lost with repeated UV irradiation. As expected, chronic UV irradiation induced significant epidermal thickening in addition to skin water loss in hairless mice, which indicates that the animal model used in this study was suitable for assessing the skin protective activity of candidate food materials.

Lactoferrin is an iron-binding glycoprotein, and it exerts various host defensive effects, not only in newborn infants but also in adult animals and humans. It is synthesized by glandular epithelial cells and is secreted in body fluids, such as milk, colostrum, tears, saliva, and mucosal secretions (Brock, 2002).

Previous animal studies have demonstrated that the administration of LF exhibited antiinflammatory activity through inhibition of the production of proinflammatory cytokines. Both intradermal injection 

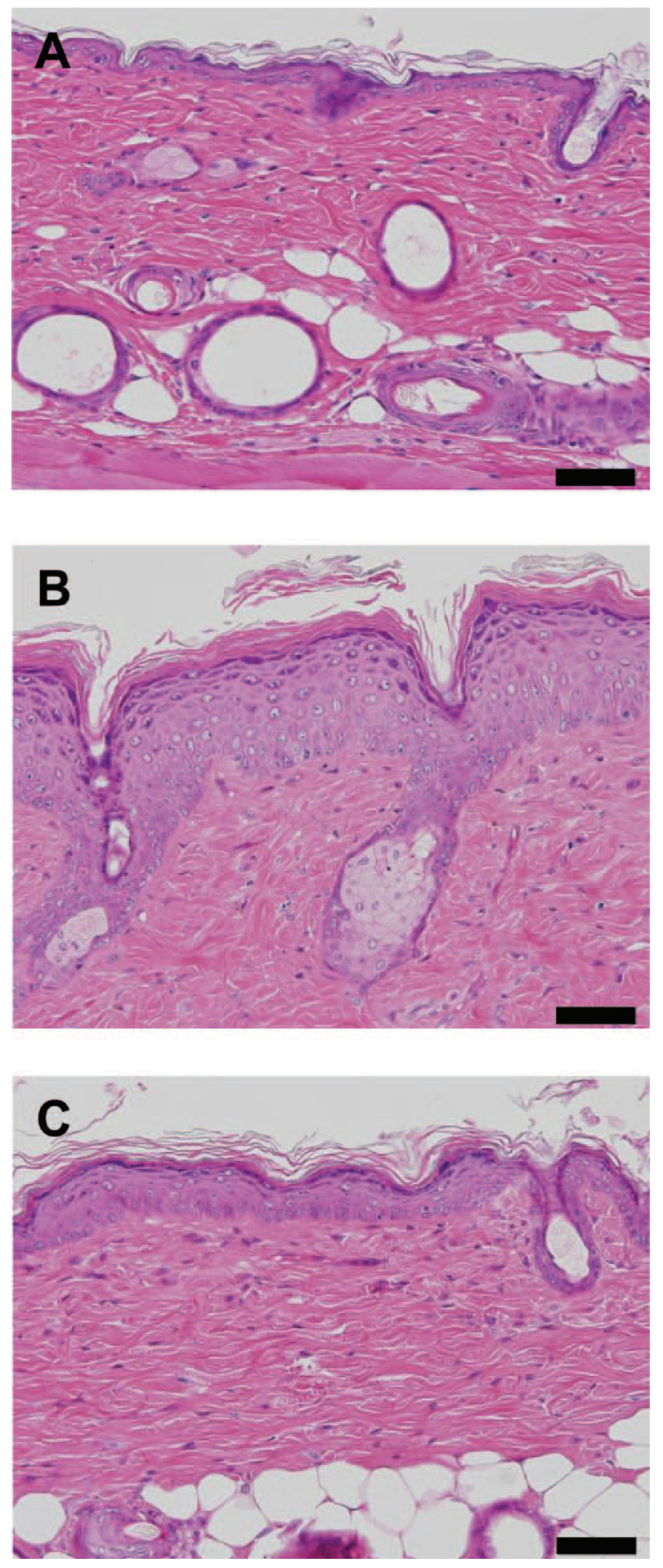

Figure 3. Histology of UVB-irradiated skin in hairless mouse, visualized with hematoxylin and eosin staining. (A) UV(-) Saline, nonirradiated mouse; (B) UV(+) Saline, UVB-irradiated mouse; (C) $\mathrm{UV}(+) \mathrm{LF}$, UVB-irradiated and lactoferrin (LF)-administered mouse. Bar size $=50 \mu \mathrm{m}$. and topical administration of LF have been shown to inhibit the allergen-induced or IL-1 $\beta$-induced migration of epidermal Langerhans cells and the accumulation of dendritic cells in the lymph nodes by controlling the local production of TNF- $\alpha$ (Cumberbatch et al., 2000). In acute dextran sulfate-induced colitis mice, orally administered LF significantly diminished IL-1 $\beta$ levels in the serum and the crypt score (Håversen et al., 2003). The oral administration of LF has been shown to attenuate LPS-stimulated adjuvant arthritis in rats with the downregulation of TNF- $\alpha$ and upregulation of IL-10 (Hayashida et al., 2004). In intestinal ischemiareperfusion injury rats, serum levels of TNF- $\alpha$, IL-1 $\beta$, and IL- 6 were decreased and injury was improved by the oral supplementation of LF (Zhang et al., 2012). Moreover, LF was shown to exert an immunomodulatory effect via the intestinal immune system. Kuhara et al. (2012) reported that orally administered LF exhibited an antiinflammatory effect on hepatic and colitis mouse models by increasing the production of IL-11 and bone morphogenetic protein 2 in the intestine.

Using this photoaging model, we first presented the protective activity of orally administered LF against UV-irradiated skin damage, including a reduction in TEWL, increase in skin hydration, and suppression of epidermal hyperplasia. In addition, we performed the same photodamage study at LF administration doses of 200 and $800 \mathrm{mg} / \mathrm{kg}$ per day. These results showed a reduction in TEWL and suppression of epidermal hyperplasia at $200 \mathrm{mg} / \mathrm{kg}$ per day (data not shown). Ultraviolet B damages not only the epidermis, but also the whole skin. The reduction in TEWL indicated an improvement in epidermal barrier function and dermal composition. The increase in skin hydration was considered to be the result of an improvement in metabolism in both the epidermis and dermis.

Other studies have shown that dietary supplementation with antioxidant ingredients such as green tea polyphenols and carotenoids had skin-protective activities in photodamage mice models (González et al., 2003; Vayalil et al., 2004). Supplementation with these antioxidant ingredients inhibits aberrant epidermal hyperplasia, protein oxidation, and matrix metalloproteinase induction in UV-irradiated mouse skin. In this study, we demonstrated the photoprotective activity of LF supplementation. However, orally supplemented LF may exert its effect in a different manner from that of the antioxidant ingredients.

First, to identify the protective mechanism of LF, we analyzed ROS-related factors in the skin. No significant differences in SOD-like activity or MDA levels were observed in the skin between the UV(-) Saline and UV(+) Saline groups. This result seems to indicate that SOD-like activity, one of the representative 


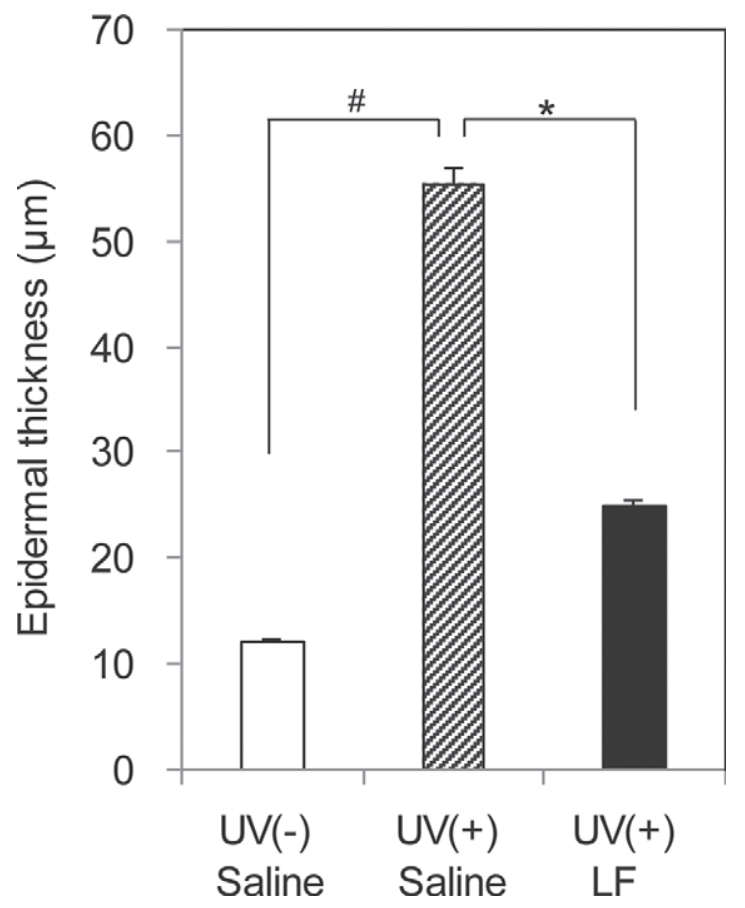

Figure 4. Epidermal thickness of UVB-irradiated skin in hairless mouse. $\mathrm{UV}(-)$ Saline $=$ nonirradiated group; $\mathrm{UV}(+)$ Saline $=$ UVB-irradiated group; UV(+) LF, UVB-irradiated and lactoferrin (LF)-administered group. ${ }^{*} P<0.05$ significant difference between the $\mathrm{UV}(+)$ Saline group and UV $(+)$ LF group. \#significant $(P<0.05)$ difference between the UV $(-)$ Saline group and UV $(+)$ Saline group; *significant $(P<0.05)$ difference between the UV $(+)$ Saline group and $\mathrm{UV}(+)$ LF group. Epidermal thickness was evaluated at 30 points per mouse, and the average thickness of 8 mice was adopted.

antioxidization activities, participates little in the skinprotective activity of LF.

Second, to address the effect of orally administered LF in regulating UV-induced cutaneous inflammatory, we analyzed inflammatory cytokine levels, including IL-1 $\beta$, IL- 6 , and TNF- $\alpha$, in the skin and serum of mice with and without UV irradiation. We found that LF supplementation significantly suppressed the UV-induced increase in IL-1 $\beta$ levels in the skin and epidermal hyperplasia. Interleukin- $1 \beta$ is considered to induce epidermal Langerhans cell migration in the early stage of the immune response (Enk et al., 1993). It has been shown that Langerhans cells found suprabasally in the epidermis can sense UV damage directly or indirectly and are responsible for generating IL-1 1 , leading to inflammasome activation, which contributes to the induction of tolerance, the promotion of chronic inflammation, and ultimate progression of neoplasia in response to UV exposure (Nasti and Timares, 2012). Orally supplemented LF is considered to exhibit antiinflammatory and immunomodulative effects directly or indirectly via the intestinal immune system in response to UV irradiation.

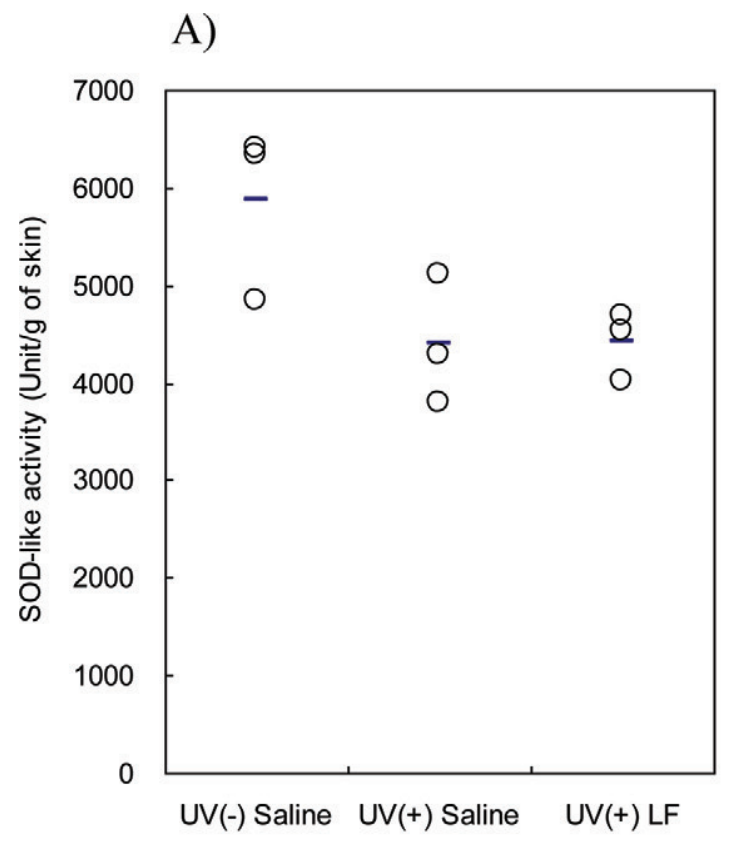

B)

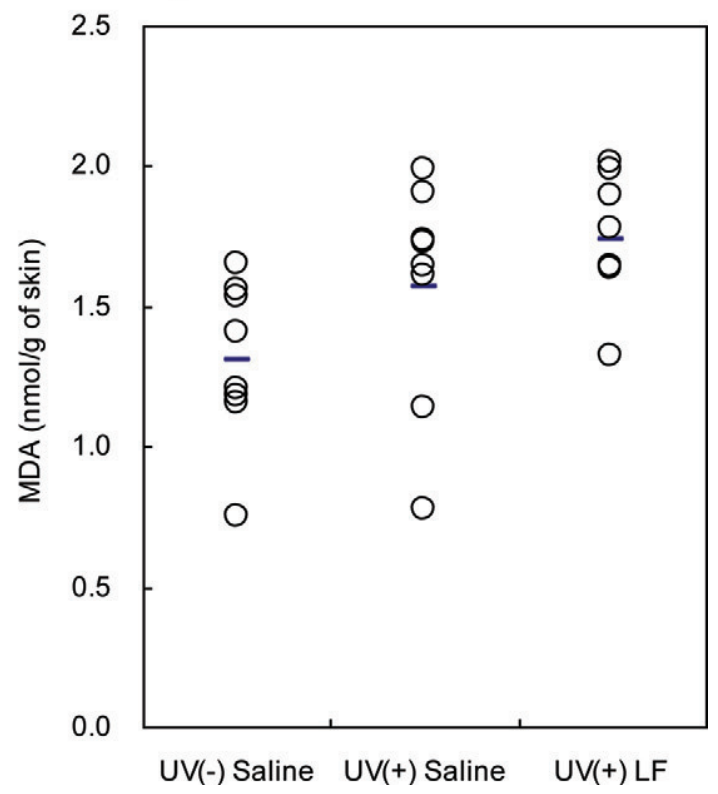

Figure 5. Effect of oral administration of lactoferrin (LF) on antioxidative parameters in the skin of UVB-irradiated hairless mice. $\mathrm{UV}(-)$ Saline $=$ nonirradiated group; $\mathrm{UV}(+)$ Saline $=$ UVB-irradiated group; $\mathrm{UV}(+) \mathrm{LF}$, UVB-irradiated and lactoferrin (LF)-administered group. (A) Superoxide dismutase (SOD)-like activity in the skin ( $\mathrm{n}=$ 3); (B) malondialdehyde (MDA) levels in the skin $(\mathrm{n}=8)$. Data are presented as the mean \pm SEM. Color version available in the online PDF.

Regarding protein hydrolysates, Tanaka et al. (2009) confirmed that orally administered collagen peptides improved skin hydration, and attenuated epidermal hyperplasia in the same UV-induced photodamage model. 


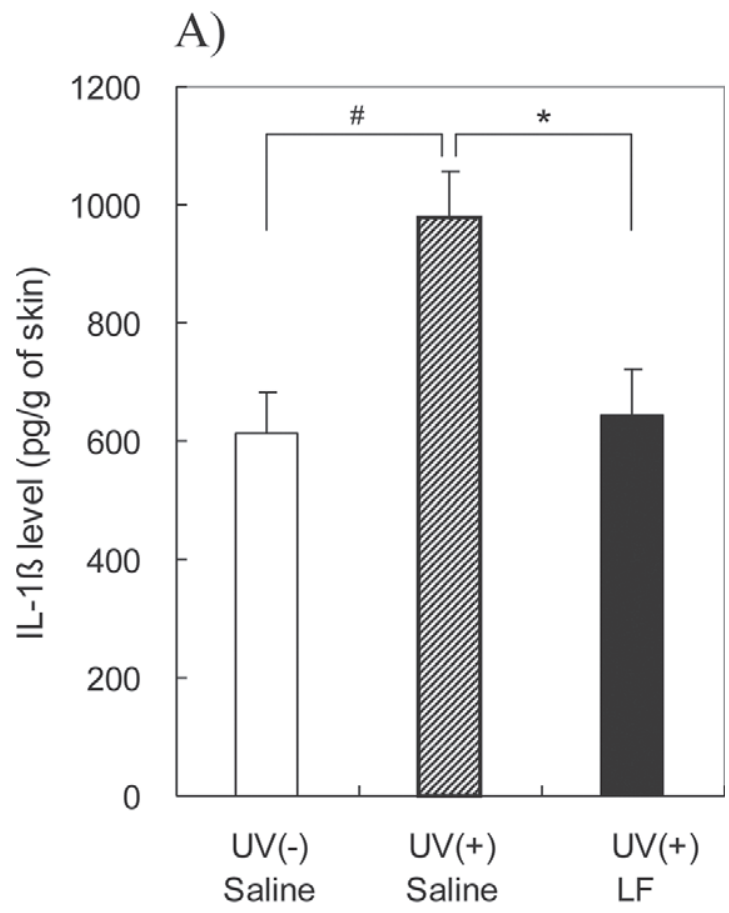

B)

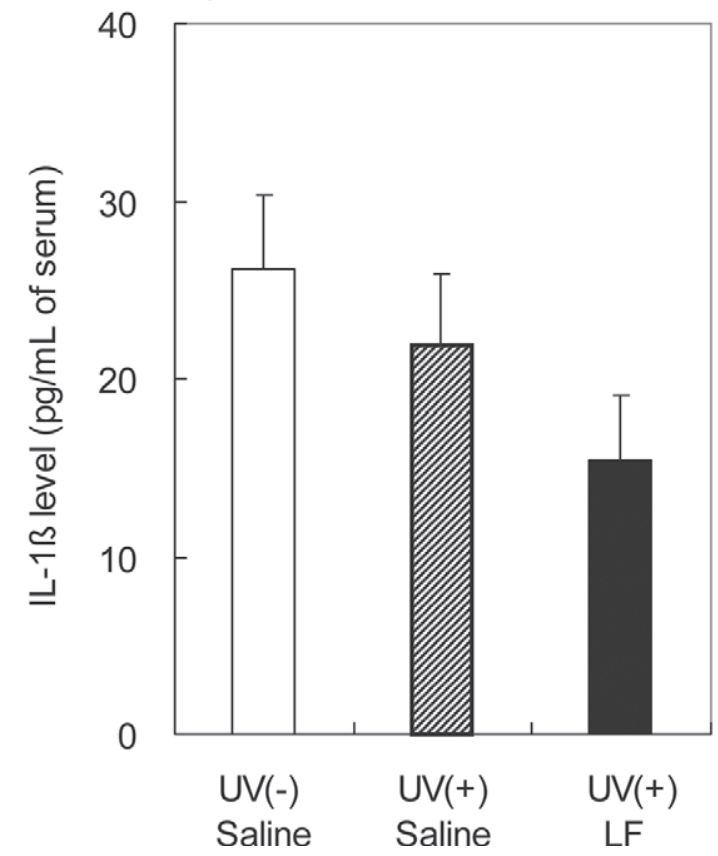

Figure 6. Effect of oral administration of lactoferrin (LF) on IL-1 $\beta$ levels in (A) mouse skin, and (B) serum. UV(-) Saline = nonirradiated group; UV $(+)$ Saline = UVB-irradiated group; UV $(+)$ LF, UVBirradiated and lactoferrin (LF)-administered group. Data are presented as the mean \pm SEM; \#significant $(P<0.05)$ difference between the $\mathrm{UV}(-)$ Saline group and UV(+) Saline group; *significant $(P<0.05)$ difference between the UV(+) Saline group and UV $(+)$ LF group.
Because collagen peptides are found in the peripheral blood after oral administration, they have been suggested to have a direct influence on the dermis.

Although our results indicate that LF has an antiinflammatory effect on UV-damaged skin, orally administered LF absorbed via the intestinal tract may not directly reach the skin because LF was not detected in blood after its administration in a previous study. Other possible mechanisms remain for the photoprotective function of LF. Lactoferrin may modulate UVinduced inflammatory and oxidative signals in the skin in an indirect manner, which may attenuate abnormal epidermal hyperplasia and apoptotic cells and significantly improve TEWL and skin hydration.

\section{CONCLUSIONS}

Our findings indicate, for the first time, that oral supplementation with LF has the potential to inhibit UV-stimulated IL-1 $\beta$ production and prevent epidermal barrier dysfunction. Further studies are needed to elucidate the relationship between the antiinflammatory effects and skin-protective function of LF.

\section{ACKNOWLEDGMENTS}

The authors thank Azusa Tanaka (Morinaga Milk Industry, Kanagawa, Japan) for her technical support with the histological analysis.

\section{REFERENCES}

Actor, J. K., S. A. Hwang, and M. L. Kruzel. 2009. Lactoferrin as a natural immune modulator. Curr. Pharm. Des. 15:1956-1973.

Baker, E. N. 2005. Lactoferrin: A multi-tasking protein par excellence. Cell. Mol. Life Sci. 62:2529-2530.

Bellamy, W., M. Takase, H. Wakabayashi, K. Kawase, and M. Tomita. 1992. Antibacterial spectrum of lactoferricin B, a potent bactericidal peptide derived from the N-terminal region of bovine lactoferrin. J. Appl. Bacteriol. 73:472-479.

Berneburg, M., N. Gattermann, H. Stege, M. Grewe, K. Vogelsang, T. Ruzicka, and J. Krutmann. 1997. Chronically ultraviolet-exposed human skin shows a higher mutation frequency of mitochondrial DNA as compared to unexposed skin and the hematopoietic system. Photochem. Photobiol. 66:271-275.

Brock, J. H. 2002. The physiology of lactoferrin. Biochem. Cell Biol. $80: 1-6$.

Cumberbatch, M., R. J. Dearman, S. Uribe-Luna, D. R. Headon, P. P. Ward, O. M. Conneely, and I. Kimber. 2000. Regulation of epidermal Langerhans cell migration by lactoferrin. Immunology 100:21-28.

Enk, A. H., V. L. Angeloni, M. C. Udey, and S. I. Katz. 1993. An essential role for Langerhans cell-derived IL-1 $\beta$ in the initiation of primary immune responses in skin. J. Immunol. 150:3698-3704.

Fisher, G. J., S. Datta, Z. Wang, X. Y. Li, T. Quan, J. H. Chung, S. Kang, and J. J. Voorhees. 2000. c-Jun-dependent inhibition of cutaneous procollagen transcription following ultraviolet irradiation is reversed by all-trans retinoic acid. J. Clin. Invest. 106:663-670.

Fisher, G. J., S. Kang, J. Varani, Z. Bata-Csorgo, Y. Wan, S. Datta, and J. J. Voorhees. 2002. Mechanisms of photoaging and chronological skin aging. Arch. Dermatol. 138:1462-1470. 
Freiburghaus, C., H. Lindmark-Månsson, M. Paulsson, and S. Oredsson. 2012. Reduction of ultraviolet light-induced DNA damage in human colon cancer cells treated with a lactoferrin-derived peptide. J. Dairy Sci. 95:5552-5560.

Fujihara, T., T. Nagano, K. Endo, M. Nakamura, and K. Nakata. 2000. Lactoferrin protects against UV-B irradiation-induced corneal epithelial damage in rats. Cornea 19:207-211.

González, S., S. Astner, W. An, D. Goukassian, and M. A. Pathak. 2003. Dietary lutein/zeaxanthin decreases ultraviolet B-induced epidermal hyperproliferation and acute inflammation in hairless mice. J. Invest. Dermatol. 121:399-405.

Håversen, L. A., L. Baltzer, G. Dolphin, L. A. Hanson, and I. MattsbyBaltzer. 2003. Anti-inflammatory activities of human lactoferrin in acute dextran sulphate-induced colitis in mice. Scand. J. Immunol. 57:2-10.

Hayashida, K., T. Kaneko, T. Takeuchi, H. Shimizu, K. Ando, and E. Harada. 2004. Oral administration of lactoferrin inhibits inflammation and nociception in rat adjuvant-induced arthritis. J. Vet. Med. Sci. 66:149-154.

Iizawa, O., T. Kato, H. Tagami, H. Akamatsu, and Y. Niwa. 1994 Long-term follow-up study of changes in lipid peroxide levels and the activity of superoxide dismutase, catalase and glutathione peroxidase in mouse skin after acute and chronic UV irradiation. Arch. Dermatol. Res. 286:47-52.

Kiss, I., S. Chen, and K. M. Tramposch. 1991. The effect of high and low ultraviolet-B dose exposure on the degree of hairless mouse skin wrinkling. Photochem. Photobiol. 53:109-112.

Kligman, L. H., F. J. Akin, and A. M. Kligman. 1982. Prevention of ultraviolet damage to the dermis of hairless mice by sunscreens. J. Invest. Dermatol. 78:181-189.

Kuhara, T., K. Yamauchi, and K. Iwatsuki. 2012. Bovine lactoferrin induces interleukin-11 production in a hepatitis mouse model and human intestinal myofibroblasts. Eur. J. Nutr. 51:343-351.

Legrand, D. 2012. Lactoferrin, a key molecule in immune and inflammatory processes. Biochem. Cell Biol. 90:252-268.

Meffert, H., W. Diezel, and N. Sönnichsen. 1976. Stable lipid peroxidation products in human skin: Detection, ultraviolet light-induced increase, pathogenic importance. Experientia 32:1397-1398.

Nasti, T. H., and L. Timares. 2012. Inflammasome activation of IL-1 family mediators in response to cutaneous photodamage. Photochem. Photobiol. 88:1111-1125.

Okada, K., Y. Takahashi, K. Ohnishi, O. Ishikawa, and Y. Miyachi. 1994. Time-dependent effect of chronic UV irradiation on superoxide dismutase and catalase activity in hairless mice skin. J. Dermatol. Sci. 8:183-186.

Pence, B. C., and M. F. Naylor. 1990. Effects of single-dose ultraviolet radiation on skin superoxide dismutase, catalase, and xanthine oxidase in hairless mice. J. Invest. Dermatol. 95:213-216.

Quan, T., T. He, S. Kang, J. J. Voorhees, and G. J. Fisher. 2004. Solar ultraviolet irradiation reduces collagen in photoaged human skin by blocking transforming growth factor-beta type II receptor/ Smad signaling. Am. J. Pathol. 165:741-751.

Rittié, L., and G. J. Fisher. 2002. UV-light-induced signal cascades and skin aging. Ageing Res. Rev. 1:705-720.

Saito, S., Y. Takayama, K. Mizumachi, and C. Suzuki. 2011. Lactoferrin promotes hyaluronan synthesis in human dermal fibroblasts. Biotechnol. Lett. 33:33-39.
Sander, C. S., H. Chang, S. Salzmann, C. S. Müller, S. EkanayakeMudiyanselage, P. Elsner, and J. J. Thiele. 2002. Photoaging is associated with protein oxidation in human skin in vivo. J. Invest. Dermatol. 118:618-625.

Schwartz, E. 1988. Connective tissue alterations in the skin of ultraviolet irradiated hairless mice. J. Invest. Dermatol. 91:158-161.

Shimmura, S., M. Suematsu, M. Shimoyama, K. Tsubota, Y. Oguchi, and Y. Ishimura. 1996. Subthreshold UV radiation-induced peroxide formation in cultured corneal epithelial cells: The protective effects of lactoferrin. Exp. Eye Res. 63:519-526.

Shindo, Y., E. Witt, D. Han, and L. Packer. 1994. Dose-response effects of acute ultraviolet irradiation on antioxidants and molecular markers of oxidation in murine epidermis and dermis. J. Invest. Dermatol. 102:470-475.

Takayama, Y., and R. Aoki. 2012. Roles of lactoferrin on skin wound healing. Biochem. Cell Biol. 90:497-503.

Takayama, Y., and T. Takezawa. 2006. Lactoferrin promotes collagen gel contractile activity of fibroblasts mediated by lipoprotein receptors. Biochem. Cell Biol. 84:268-274.

Tanaka, M., Y. Koyama, and Y. Nomura. 2009. Effects of collagen peptide ingestion on UV-B-induced skin damage. Biosci. Biotechnol. Biochem. 73:930-932.

Tang, L., T. Cui, J. J. Wu, W. Liu-Mares, N. Huang, and J. Li. 2010a. A rice-derived recombinant human lactoferrin stimulates fibroblast proliferation, migration, and sustains cell survival. Wound Repair Regen. 18:123-131.

Tang, L., J. J. Wu, Q. Ma, T. Cui, F. M. Andreopoulos, J. Gil, J. Valdes, S. C. Davis, and J. Li. 2010b. Human lactoferrin stimulates skin keratinocyte function and wound re-epithelialization. Br. J. Dermatol. 163:38-47.

Vayalil, P. K., A. Mittal, Y. Hara, C. A. Elmets, and S. K. Katiyar 2004. Green tea polyphenols prevent ultraviolet light-induced oxidative damage and matrix metalloproteinases expression in mouse skin. J. Invest. Dermatol. 122:1480-1487.

Wan, Y., A. Belt, Z. Wang, J. Voorhees, and G. Fisher. 2001. Transmodulation of epidermal growth factor receptor mediates IL-1 $\beta$-induced MMP-1 expression in cultured human keratinocytes. Int. J. Mol. Med. 7:329-334.

Yaar, M., M. S. Eller, and B. A. Gilchrest. 2002. Fifty years of skin aging. J. Investig. Dermatol. Symp. Proc. 7:51-58.

Yamauchi, K., H. Wakabayashi, S. Hashimoto, S. Teraguchi, H. Hayasawa, and M. Tomita. 1998. Effects of orally administered bovine lactoferrin on the immune system of healthy volunteers. Adv. Exp. Med. Biol. 443:261-265.

Yan, D. D. Chen, J. Shen, G. Xiao, A. J. van Wijnen, and H. J. Im. 2013. Bovine lactoferricin is anti-inflammatory and anti-catabolic in human articular cartilage and synovium. J. Cell. Physiol. 228:447-456.

Zhang, T., Y. Wang, R. Ban, L. Tong, H. Qiao, H. Lao, H. Zhao, X. Jiang, X. Sun, and F. Zhang. 2012. Oral administration of lactoferrin attenuates intestinal ischemia-reperfusion injury in rats. Eur. Surg. Res. 49:99-106.

Zheng, P., and L. H. Kligman. 1993. UVA-induced ultrastructural changes in hairless mouse skin: A comparison to UVB-induced damage. J. Invest. Dermatol. 100:194-199. 